Article

\title{
Evaluating Methodology for the Service Extent of Refugee Parks in Changchun, China
}

\author{
Xiaoling Li ${ }^{1,2}$, Chunliang Xiu ${ }^{3} \mathbb{D}$, Ye Wei ${ }^{1} \mathbb{D}$ and Hong S. He ${ }^{4, *}$ \\ 1 School of Geographical Sciences, Northeast Normal University, Changchun 130024, China; \\ lix1027@nenu.edu.cn (X.L.); weiy742@nenu.edu.cn (Y.W.) \\ 2 Key Laboratory of Geographical Processes and Ecological Security in Changbai Mountains, Ministry of \\ Education, Changchun 130024, China \\ 3 College of Jang Ho Architecture, Northeastern University, Shenyang 110169, China; \\ xiuchunliang@mail.neu.edu.cn \\ 4 School of Natural Resources, University of Missouri, Columbia, MO 65211, USA \\ * Correspondence: heh@missouri.edu
}

Received: 16 June 2020; Accepted: 10 July 2020; Published: 16 July 2020

\begin{abstract}
Refugee parks are general parks that can serve as emergency shelters in cities. The core issue of refugee parks lies in their service extent they provided. Globally, the service extent of refugee parks is determined by the Euclidean or actual road network distance methods. The former lacks measurement accuracy, whereas the latter lacks the consideration of human dimension and proximity. Hence, we propose the nearest neighbor method, which considers not only the locations of refugee parks and sub-districts, but also road networks and census data. Using this method, we evaluated the service extent of refugee parks in Changchun, northern China. We compared our results with the Euclidean distance method. Results showed that the nearest neighbor method effectively accounted for the effect of road network resistance and results aligned with the refuge needs of residents. Differences in both methods were mainly affected by the size of the parks and local road network and population densities. The Euclidean approach determines the service extent based on a unified service radius, thus producing greater errors. The nearest neighbor method can reveal the spatial imbalance of refugee parks, as well as the mismatch between the park size and population distribution. Furthermore, the nearest neighbor method implements policies of spatial optimization of urban refugee parks. As a general method, it should be suited to different types of disasters.
\end{abstract}

Keywords: nearest neighbor method; gaussian-based two-step floating catchment area method; spatial layout of refugee parks; network analyst

\section{Introduction}

Large cities often have an abundance of resources and development opportunities. They are often entangled by high population densities and scarce natural spaces. Thus, large cities are particularly vulnerable to disasters and accidents [1], such as rainstorms, heavy snow, fires, earthquakes, toxic gas leakage, and tsunamis. Moreover, they also suffer the impact of "urban diseases", such as traffic jams, air pollution, and water pollution. These problems are not as pronounced in small cities but can jeopardize large cities. For safe urban environments, natural spaces should not only satisfy the needs of leisure and recreation, but also serve as effective places of disaster prevention and rescue.

Most developed countries with frequent natural disasters have developed and implemented the concept of urban disaster prevention green spaces [2-5]. The implementation of the City Park Law in Japan (1993) was the first government formulated a policy that placed city parks in the status of refugee parks [6]. Refugee parks refer to the urban green spaces that perform the following functions: 
(1) Satisfy the leisure needs of residents during ordinary time, providing recreation, sightseeing, and cultural entertainment; (2) provide shelters and emergency rescue for residents in case of disasters; and (3) mitigate or prevent the spread of disasters and potential secondary disasters [7]. Refugee parks are designed to assure fire prevention, evacuation, and flood discharge, so that they are essential physical spaces for urban safety. Urban planning, especially in large cities, must consider refugee parks as emergency shelters in order to provide residents with safe and practical places in the event of a severe disaster [8]. Emergency response and evacuation time of city residents are key to public security and safety when natural disasters occur $[9,10]$. Remote sensing and geographic information systems (GIS) are also important tools in this area [11]. In combination with real-time ground data and statistic data, remote sensing and GIS can and simulate scenarios of affected population and locations under disasters and provide visualization to aid decision making [12-14].

The core issue of refugee parks lies in their service extent for disaster prevention and alleviation, namely, the spatial extent of planned or actual refuge services by a park. If the service extent of a disaster prevention park is too large, it will take a long time for the evacuees to reach the refugee park, thus increasing the risk especially to the elderly, children, pregnant women, and the disabled $[15,16]$. It is important to evaluate the service extent for refugee parks to ensure the safety of the evacuees, install comprehensive urban disaster prevention systems, and respond to anthropogenic and natural disasters. Accessibility is an important indicator that measures the relationship between the service extent of refugee parks and the refugee population. However, park accessibility and its service extent are currently measured using the methods that differ greatly [17].

Globally, the spatial accessibility of refugee parks is predominantly evaluated based on the Euclidean or actual road network distance methods. The measurement methods that calculate the Euclidean distance include the Gaussian-based two-step approach [18], convenience sampling method [19], Thiessen polygon method [20], and space syntax method [21]. The Euclidean distance method assumes that the refugee population and refugee parks are evenly distributed, and the service radius of a refugee park can be set manually as a straight radius between the residential districts and the park. This method is intuitive and highly practical, but it ignores the physical resistance (including artificial landscape elements like buildings and natural obstacles like rivers) encountered by the evacuees trying to reach the target refugee parks. To overcome these issues, scholars proposed actual road network distance methods, namely, incorporating the traffic-flow information on evacuation routes and measuring park accessibility by estimating the duration of refugee evacuation to refugee parks [22]. Such existing methods include a simple calculation method that considers road network factors [3] and a gravity model method [23]. Actual road network distance methods overcome the accessibility measurement issues based on the Euclidean distance and are better suited to the real scenarios of an emergency evacuation. However, spatial accessibility is the main evaluation index and is prone to ignore the spatial distribution of evacuees, thus causing a mismatch between the resource allocation and actual evacuation needs.

Currently, the methods for measuring spatial accessibility based on the Euclidean distance are used more frequently than those based on the actual road network distance. However, the attraction of disaster prevention green spaces to evacuees decreases as the distance between them increases. To determine the service extent of refugee parks, it is necessary to use an efficient method by (1) selecting the nearest refugee park based on human-oriented principles and using the Euclidean distance method, and (2) revealing the scenarios of emergency evacuation using an actual road network distance method. From the perspective of economic geography, we propose the usage of the nearest neighbor method to define the service extent of refugee parks. The method also presents an index system for evaluating the accommodation capacity of refugee parks according to the relationship between the service extent and the population within the district. The nearest neighbor method utilizes the nearest principle-that is, accessibility is measured according to the road distance to the nearest object, sub-district in this study. At present, the method has been predominantly applied to the studies of the location pattern of commercial urban retail stores [24]. The nearest neighbor method assumes that people usually 
choose to visit the nearest commercial venue. This is consistent with the assumption made by the Euclidean distance method, that is, people choose to evacuate to the nearest refugee park in case of disasters. This method can be used to determine the spatial distribution of services in a refugee park. Furthermore, the optimal spatial distribution of refugee parks can be measured using a multi-objective programming approach combining the service extent and the nearby population indices [25].

Changchun is a major city in northeast China with rapid economic and population growth. From 2000 to 2015, urbanization increase from about $40 \%$ to $60 \%$, while built-up land increased from $394 \mathrm{~km}^{2}$ to $471 \mathrm{~km}^{2}$ [26]. Its current population is 442.5 million [27], and both its population and building density continue to grow. Historically, Changchun has been affected by natural disasters [28]. Its natural conditions are relatively homogeneous with flat topography and absent large natural barriers, such as high mountains or large rivers. The urban road network is a typical square grid network. Compared to a curved road network, a regular straight-line network is more straightforward for determining the spatial service extent of refugee parks. Changchun has many common features shared by the cities of northern China and is suitable to be studied using the nearest neighbor method. As exemplified by Changchun City, China, the nearest neighbor method is applied to the study of the service extent of refugee parks. The objective of this study is to explore the applicability of the nearest neighbor method to the studies of the accessibility of disaster prevention green spaces. Specifically, we intend to answer the following questions: (1) Can the nearest neighbor method be applied to determining the spatial service extent of refugee parks?; (2) are the evaluation results obtained by the nearest neighbor method more suitable for evaluating the spatial layout of refugee parks?; and (3) is it possible to combine the service extent with the population indices to evaluate the rationality of the spatial distribution of refugee parks? We answer these questions by comparing the results of our method with the of the most commonly used Gaussian-based two-step method.

\section{Materials and Methods}

\subsection{Study Area}

Changchun is the capital city of Jilin province, China, and its political, economic, and cultural center (Figure 1). The city is located at the junction of the Zhangguangcai Ridge (a branch of the Changbai Mountains) and the Songliao Basin. The local tectonic environment of the central urban area comprises the Xinglong Mountain anticline in the northeast and the Xixin depression in the southwest. The Tancheng-Lujiang fault zone of eastern China stretches in a northeastward direction, and its two branches pass through Changchun. According to the Seismic Ground Motion Parameter Zonation Map of China (GB18306-2001) and Code for Seismic Design of Buildings (GB50011-2001), the seismic fortification intensity of Changchun is determined to be grade 7. In recent years, Changchun has suffered from natural disasters, such as geological disasters, droughts, floods, and meteorological disasters. As stated in the notice of Changchun's comprehensive disaster prevention and mitigation plan (2016-2020) [29], recently released by the General Office of the People's Government of Changchun, the city will make full use of public service facilities (e.g., parks) to build and transform them into emergency shelters suited to local conditions. The notice also states that the nearest and convenient resettlement services for disaster-stricken people are to be provided. Population by sub-district existed in the decadal census data, which was last conducted in 2010. To ensure the 2010 population matches the current sub-regions, we restricted our study area in the core city area (also known as the old city area). Population and street networks have remained generally stable from 2010 to 2020 in the core area, while most changes were newer and taller buildings, which will not affect our analysis in this study [30]. The central urban districts in Changchun include Nanguan, Chaoyang, Kuancheng, Lvyuan, and Erdao Districts (it excludes Shuangyang and Jiutai Districts). The central urban districts are comprised of a total of 46 sub-districts. To calculate the service extent of refugee parks more accurately, unpopulated land (e.g., rivers, railway facilities) was masked out from the study area. The finalized study region 
covered a total area of 23,205 $\mathrm{km}^{2}$ (Figure 1). According to China's sixth nationwide population census in 2010, Changchun's central districts have a population of approximately three million.

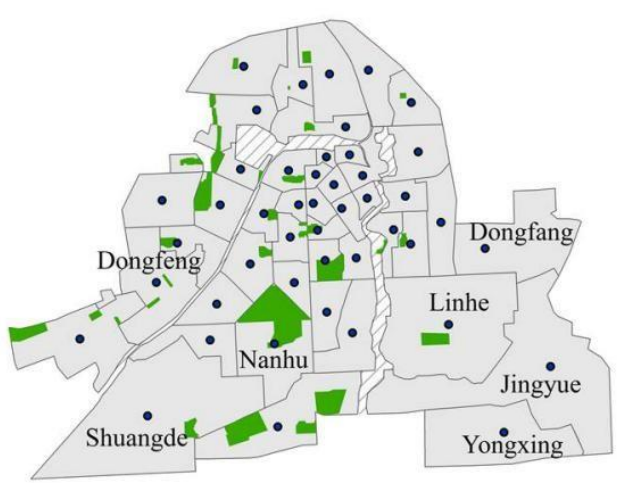

a

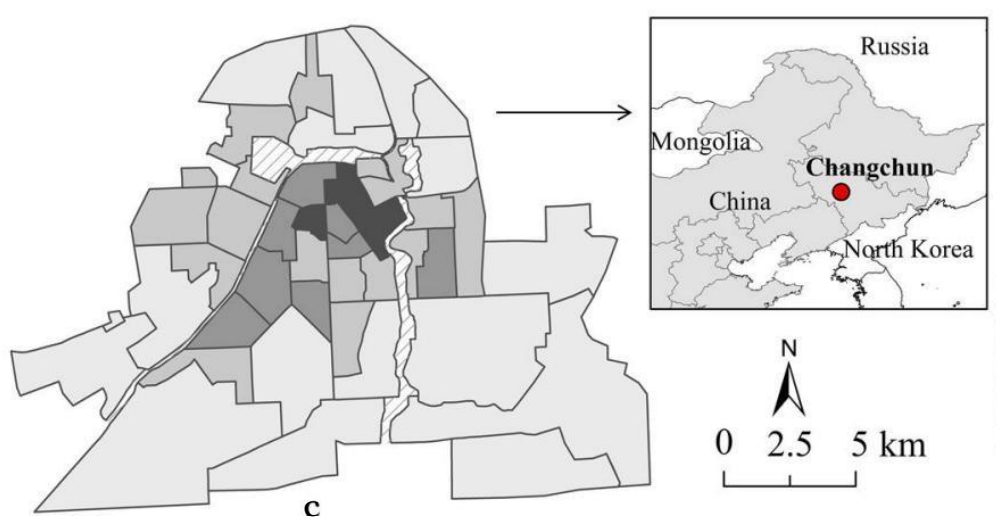

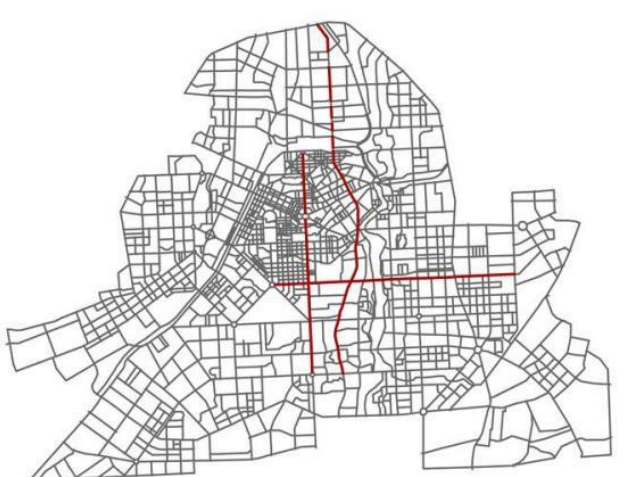

b

Figure 1. The central urban area of Changchun, China and the spatial data used in the study: (a) spatial distribution of refugee parks, (b) road network, and (c) sub-districts of the central urban area.

\subsection{General Approach}

We used multi spatial data, including remote sensing, road network vector, census population, and governmental reports. These data were compiled and inputted into the two models: Nearest neighbor method and Gaussian-based two-step floating catchment area method. The two models delineated service extent for each refugee park, and calculated the average green land area per capita. We then verified the results from the nearest neighbor method against the field data and compared the delineated service extents and the average green area per capita from two models. By doing so, we addressed the three specific objectives identified in the study (Figure 2). 
Spatial data processing: Image, Census population, Open Street Map, Policy, document, field survey data

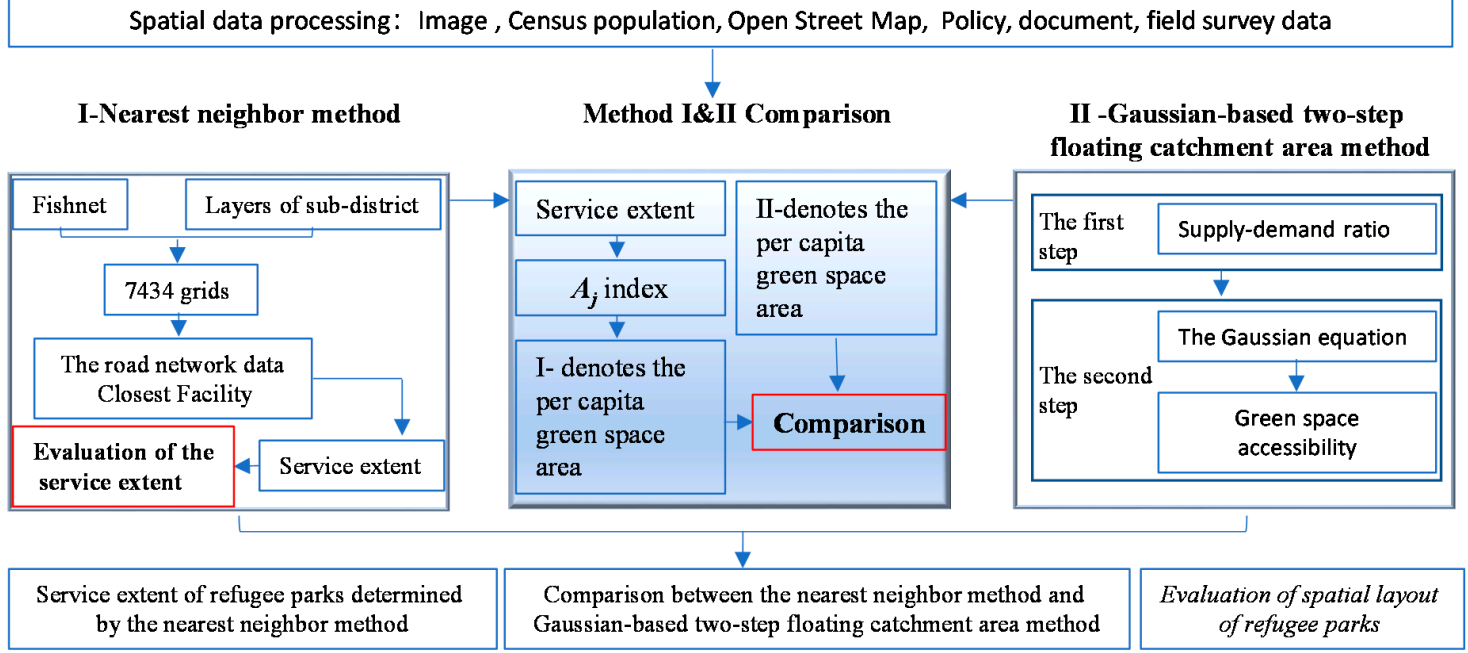

Figure 2. The general approach for evaluating the methodologies of determining the service extent of refugee parks in Changchun, China.

\subsection{Data}

Together with the field survey data, the basic data from the Ecological Green Space System Planning for Building a Green and Livable Forest City in Changchun (2013-2030) were imported into ArcGIS by extracting the coordinates via GoogleEarth. The digital map layers of the refugee parks in Changchun were digitized (Figure 1a). The road network data of the central urban area in Changchun were obtained through the Open Street Map of ArcGIS (Figure 1b). The residential data of sub-districts were obtained from China's sixth population census data of 2010 (Figure 1c). Sub-district boundary data were acquired from the Bureau of Civil Affairs of Changchun city. At present, Changchun has a total of 33 urban parks with an area of at least $1 \mathrm{hm}^{2}$ that may be converted to refugee parks. Overall, there is no unified refugee park system in the central urban area of Changchun, due to the high population density, uneven distribution of parks and green spaces, and the lack of designation of refugee parks [31].

Parks can serve for different disaster prevention functions, depending on the size of their green space, structure, and supporting facilities. Small parks can be used as emergency refugee parks, medium ones can be used as designated disaster prevention parks, and the large ones can be used as central refugee parks. Existing studies place special emphasis on the disaster prevention functions of urban parks and argue that all parks with an area of at least $1 \mathrm{hm}^{2}$ should be transformed into refugee parks [32]. According to the published studies [33], refugee parks are classified as: (1) Central refugee parks representing city-level or district-level parks with an area of at least $50 \mathrm{hm}^{2}$, service radius of approximately $5 \mathrm{~km}$, and the greatest accommodation capacity; (2) designated refugee parks with an area of at least $10 \mathrm{hm} 2$, located within $1 \mathrm{~h}$ walking time, and a service radius of $2-3 \mathrm{~km}$; they can be used as shelters for district residents during a disaster and as living places for refugees within a certain period after a disaster; (3) emergency refugee parks with an area of at least $1 \mathrm{hm}^{2}$ and a service radius of 300-500 m; they can be used as emergency shelters within the first $3 \mathrm{~min}$ after the occurrence of a disaster. According to the refuge practices in China and abroad, the effective refuge area of central and designated refugee parks should be more than $2 \mathrm{~m}^{2}$ per capita, whereas the emergency refugee park should have at least $1 \mathrm{~m}^{2}$ per capita [34]. Flat space in an emergency refugee park should occupy at least $60 \%$ of its total area, while flat space in a designated or central refugee park should be at least $70 \%$ of its total area. Accordingly, researchers can determine the effective refuge area for each refugee park and calculate its population accommodation capacity [35]. 


\subsection{Nearest Neighbor Method}

The main principles of the nearest neighbor method are (1) to search for the nearest refugee facilities based on road networks using the GIS network analysis tools (e.g., ArcGIS Network Analyst); and (2) to determine the service extent of disaster prevention spaces by merging the spatial units that share the same facilities. The method includes the following basic steps. First, we refined the map layers of sub-district boundaries using the Fishnet tool in ArcGIS, divided a sub-district into multiple $200 \times 200 \mathrm{~m}$ grids, merged and clipped the grids with the map layer of a sub-district, and divided each sub-district into $0.04 \mathrm{~km}^{2}$ or smaller grids (a total of 7434 in this study). Polygons smaller than $0.04 \mathrm{~km}$, were generated within sub-district boundaries, and were used as basic study units. Then, we then create network datasets using ArcGIS Network Dataset tool based on the road network data. We used the Closest Facility tool in Arc Toolbox to identify the nearest refugee park away from each central fishnet point. Finally, we merged all of the nearest grids that shared the same refugee parks to derive the service extent of each refugee park.

\subsection{Gaussian-Based Two-Step Floating Catchment Area Method}

The Gaussian-based two-step floating catchment area method was initially used to measure the spatial accessibility of medical treatment ${ }^{4}$. It has become an important method since then, and has been used in many studies. This method assumes that accessibility is equal within a certain radius. In reality, accessibility decreases as distance increases. Subsequently, Luo and other (2003) made improvements in the method, and Dai (2011) developed an upgraded version. The Gaussian-based two-step floating catchment area method can be used to evaluate the accessibility of green spaces following two steps [12,36]:

$$
R_{j}=\frac{S_{j}}{\sum_{k \in\left\{d_{k j} \leq d_{0}\right\}} G\left(d_{k j}, d_{0}\right) P_{K}}{ }^{\prime}
$$

where $j$ is the green space, $d_{0}$ is the spatial distance threshold that generates a spatial scope (catchment); $k$ is the population of each sub-district within the spatial scope, weight is allocated by the Gaussian equation, and the weighted populations are summed up to determine the number of all potential users of green space $j$; and $R_{j}$ is the supply-demand ratio calculated by dividing the size of green space by the number of all potential users.

In the calculation equation, $p_{k}$ denotes the population of sub-district $K$ within the spatial scope $\left(d_{k j} \leq d_{0}\right)$ of green space $j ; d_{k j}$ is the spatial distance from the center of sub-district $k$ to green space $j ; s_{j}$ represents the population accommodation capacity of green space $j$ (the area of green space in this study); and $G\left(d_{k j}, d_{0}\right)$ denotes the Gaussian equation that considers spatial friction. The calculation method for the second step is as follows:

$$
G\left(d_{k j}, d_{0}\right)= \begin{cases}\frac{e^{-\left(\frac{1}{2}\right) \times\left(\frac{d_{k j}}{d_{0}}\right)^{2}}-e^{-\left(\frac{1}{2}\right)}}{1-e^{-\left(\frac{1}{2}\right)}}, & \text { if } d_{k j} \leq d_{0} \\ 0, & \text { if } d_{k j} \geq d_{0}\end{cases}
$$

where $i$ is the sub-district, $d_{0}$ is the spatial distance threshold used to generate another spatial scope; $R_{j}$ is the demand-supply ratio of each green space $l$ within the spatial scope, weight is allocated by the Gaussian equation, and the weighted demand-supply ratios $R_{j}$ are summed up to determine green space accessibility $A_{i}$ of each sub-district $i$.

$$
A_{i}=\sum_{i \in\left\{d_{k j} \leq d_{0}\right\}} G\left(d_{k j}, d_{0}\right) R_{j}
$$

where $R_{j}$ is the demand-supply ratio of green space $l$ within the spatial scope $\left(d_{k j} \leq d_{0}\right)$ of sub-district $i$. The rest of the indices are the same as those in Equation (1). 
The accessibility of green spaces in a sub-district determined by the Gaussian-based two-step floating catchment area method can be interpreted as the per capita green space area of a sub-district $\left(m^{2}\right.$ per capita in this study).

\subsection{Comparison of Methods}

To verify the utility of the nearest neighbor method for evaluating the accessibility of disaster prevention green spaces, we compared the results obtained by the Gaussian-based two-step floating catchment area and nearest neighbor methods. Since the nearest neighbor method calculated the service extent of a refugee park, whereas Gaussian-based two-step floating catchment area method calculated the per capita green space area, we used the following procedures to convert service extent to per capita green space area.

First, a conversion algorithm for index $A_{j}$ was created in order to transform the service extent per refugee park (determined by the nearest neighbor method) into the per capita green space area. As such, we superimposed the service extent of 33 refugee parks in Changchun with the map layers of 46 sub-districts. Then, we used the Clip tool in ArcGIS to clip the service extent of each refugee park along the sub-district boundaries. Finally, we summed up the green space area within the service extent of all refugee parks in each sub-district to determine the total green space area within the accessible range for each sub-district. The calculation equation used is as follows:

$$
A_{j}=\frac{\sum_{n}^{i=1} \frac{g_{1}}{G_{1}} \times s_{1}+\frac{g_{2}}{G_{2}} \times S_{2}+\cdots+\frac{g_{n}}{G_{n}} \times s_{n}}{P}(i=1,2 \ldots 33 ; j=1,2 \ldots 46),
$$

where, $g_{i}$ denotes the service extent of the $j$-th sub-district that includes the i-th refugee park; $G_{i}$ is the service extent of refugee park $i$; $s_{i}$ denotes the area of the $i$-th refugee park; $P$ denotes the total population of the $j$-th sub-district; and $A_{j}$ denotes the per capita green space area of each sub-district.

Next, according to the criteria for the per capita disaster prevention green space area [20], the per capita green space area was determined by both Gaussian-based and nearest neighbor methods. The green space per capita was eventually divided into four classes: (1) high $\left(>5 \mathrm{~m}^{2}\right)$; (2) medium-high $\left(2-5 \mathrm{~m}^{2}\right)$; (3) medium-low (1-2 $\left.\mathrm{m}^{2}\right)$; and (4) low $\left(<1 \mathrm{~m}^{2}\right)$.

Finally, we subtracted the per capita green space area determined by the Gaussian-based method from that of the nearest neighbor method to analyze the difference between the two methods. For refugee parks, the required minimum per capita green space area was established as $1 \mathrm{~m}^{2}$. According to three sets of circumstances (specifically, (1) greater than $1 \mathrm{~m}^{2}$; (2) 1 to $-1 \mathrm{~m}^{2}$; and (3) less than $-1 \mathrm{~m}^{2}$ ), the per capita green space area determined by the Gaussian-based two-step floating catchment area method may be greater than, the same as, or less than that determined by the nearest neighbor method.

\subsection{Evaluation of the Spatial Layout of Refugee Parks}

The competence of the spatial layout of refugee parks is evaluated by measuring population accommodation capacity. Population accommodation capacity refers to the ratio between the maximum population that can be accommodated by a refugee park and the residential population within its service extent. If the ratio is greater than 1 , the population accommodation capacity of a refugee park is sufficient to the refuge needs of the residents within its service extent in the event of a disaster, and its spatial layout is optimal. If the ratio is lower than 1 , the population accommodation capacity of a refugee park is insufficient and cannot satisfy the refuge needs of the residents within its service extent, i.e., its spatial layout is unsatisfactory.

The calculation procedure for the population accommodation capacity is as follows. First, we calculated the open space area of a refugee park. We assumed that the open space area of a central or designated refugee park accounts for $70 \%$ of its total area, whereas the open space area of an emergency refugee park accounts for $60 \%$ of its total area. Then, we compared the open space area of a refugee park with the per capita open space area required by disaster mitigation, thus determining the maximum 
population that can be accommodated by a park. The per capita open space area required by disaster mitigation varies with the type of the refugee park. For example, in a central or designated refugee park, the per capita open space area required by disaster mitigation is $2 \mathrm{~m}^{2}$, and for an emergency refugee park, the per capita open space area should be approximately $1 \mathrm{~m}^{2}$. Finally, we estimated the residential population within the service extent of each refugee park. We assumed that the population within each sub-district is distributed evenly. Then, the residential population within the service extent was calculated as the sum of the product of the area and the population density of all the grid cells within the service extent.

\section{Results}

\subsection{Service Extent of Refugee Parks Determined by the Nearest Neighbor Method}

The shapes of the service extent of refugee parks determined by the nearest neighbor method are similar, but not identical, to the administrative boundaries between the sub-districts (Figure 3 , Figure 1a). The service extent of refugee parks overrode the administrative boundaries. For example, the service extent of park No. 27 goes beyond the administrative boundaries of the sub-districts that it belongs to. The service extent of park No. 28 falls within the administrative boundaries of the sub-districts that it belongs to. Whereas, the service extent of park No. 22 spans the administrative boundaries of several sub-districts.

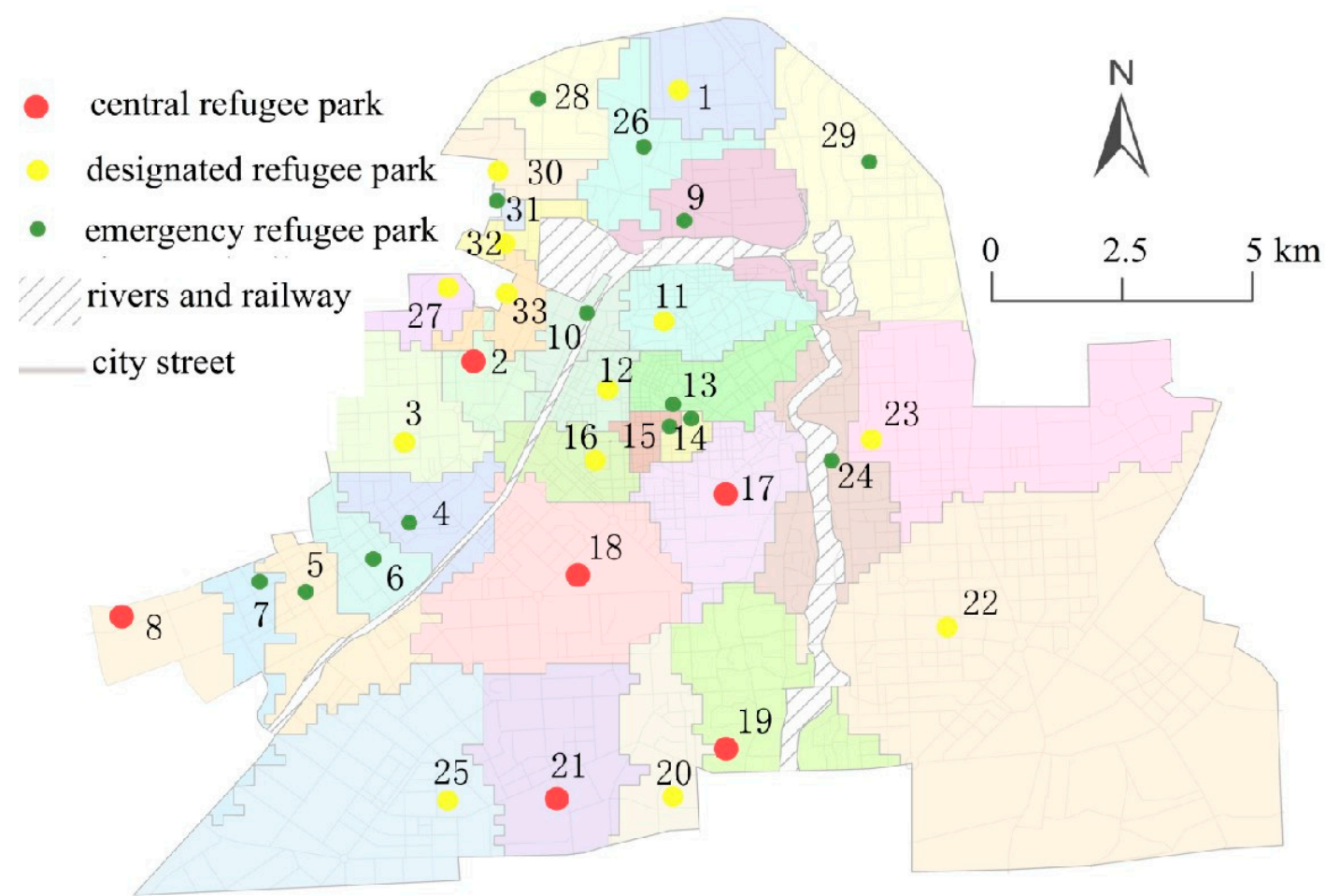

Figure 3. Map showing the distribution of different types of refugee parks in Changchun. The service extent was determined by the nearest neighbor method. Different colors denote the service extent of different refugee parks.

Central refugee parks tend to be distributed in the southwest-central urban area with a total of six parks, accounting for $18.1 \%$ of all of the Changchun's refugee parks. Designated refugee parks are mainly distributed in the northwest-central urban area with a total of 13 parks, accounting for $39.3 \%$ of all of the Changchun's refugee parks. Emergency refugee parks are scattered in the city with a total of 14 parks, accounting for $42.4 \%$ of all of the Changchun's refugee parks. The average service extent of these refugee parks is $6.83 \mathrm{~km}^{2}$. The No. 22 refugee park is located in the southeastern part of the 
central urban area and has the largest service extent of $50.92 \mathrm{~km}^{2}$, whereas the No. 31 refugee park has the smallest service extent of $0.33 \mathrm{~km}^{2}$. In total, nine refugee parks have an above-average service extent and are predominantly distributed in the central, southern, and eastern parts of Changchun. Their total service extent accounts for $63.7 \%$ of all of the Changchun's refugee parks.

The service extent is distributed unevenly across the refugee parks, or, to be more specific, is significantly polarized. The remaining 24 refugee parks have a below-average service extent and are mostly distributed in the northern and western parts of the central urban area. Overall, the service extent per refugee park tends to increase from the center to the periphery, while the service extent of refugee parks in the southern part of Changchun is greater than in any other refugee park in Changchun.

\subsection{Comparison between the Nearest Neighbor Method and Gaussian-Based Two-Step Floating Catchment Area Method}

Comparison results show that the per capita green space area determined by the two methods differs significantly, $p<0.01$ (Figure $4 \mathrm{a}, \mathrm{b}$ ). There are 20 sub-districts with the same grades of per capita green space area, which was determined by both methods, and they account for $43.5 \%$ of all of the sub-districts. Specifically, 11 sub-districts are characterized by a low grade of per capita green space area, and account for $23.9 \%$ of all of the sub-districts. Five sub-districts share a high grade of per capita green space area, and account for $10.9 \%$ of all of the sub-districts. Two sub-districts share a medium-low and low grade of per capita green space area, and account for $4.3 \%$ of all of the sub-districts (see Table 1). The per capita green space area determined by the two methods is consistent in core areas (i.e., areas surrounded by rivers, railway facilities), and is medium-high. However, the values differ significantly in the peripheral areas. In sub-districts with medium-high and high grades of per capita green space area, which was determined by the nearest neighbor method, spatial distribution reveals scattering, whereas, in the sub-districts with medium-low and low grades of per capita green space area, agglomeration can be observed (Figure 4a). On the contrary, in the sub-districts with medium-high and high grades of per capita green space area, which was determined by the Gaussian-based two-step floating catchment area method, agglomeration is present, while in the sub-districts with medium-low and low grades of per capita green space area, spatial distribution reveals scattering (Figure 4b).

Table 1. Different grades of per capita green space area determined by the nearest neighbor method and Gaussian-based two-step floating catchment area method.

\begin{tabular}{cccc}
\hline Per Capita Green Space Area & Nearest Neighbor Method & $\begin{array}{c}\text { Gaussian-Based } \\
\text { Two-Step Method }\end{array}$ & $\begin{array}{c}\text { Ratio of Coincident } \\
\text { Sub-Districts }\end{array}$ \\
\hline High $\left(>5 \mathrm{~m}^{2}\right.$ per capita) & 10 & 9 & $10.9 \%$ \\
Average $\left(2-5 \mathrm{~m}^{2}\right.$ per capita) & 12 & 5 & $4.3 \%$ \\
Medium-Low $\left(1-2 \mathrm{~m}^{2}\right.$ per capita $)$ & 10 & 6 & $4.3 \%$ \\
Low $\left(<1 \mathrm{~m}^{2}\right.$ per capita $)$ & 14 & 26 & $23.9 \%$ \\
\hline
\end{tabular}

We subtracted the per capita green space area determined by the two methods. There are 12 sub-districts with a medium-high grade of per capita green space area determined by the Gaussian-based two-step floating catchment area method with an average area of $3.74 \mathrm{~km}^{2}$. In these sub-districts, the per capita green space area is high or medium-high, the refugee parks are predominantly the central refugee parks, and relatively high population and road network densities are present. There are 17 sub-districts with a medium-low grade of per capita green space area determined by the Gaussian-based two-step floating catchment area method with an average area of $7.24 \mathrm{~km}^{2}$. In these sub-districts, the per capita green space area is low or medium-low, the refugee parks are typified as designated and emergency parks, and there are relatively low population and road network densities. Sub-districts with a consistent per capita green space area determined by both methods are mostly located in the peripheral areas of eastern Changchun. As such, their per capita green space area is low (Figure 4c). This is overall consistent with the above-mentioned results. 


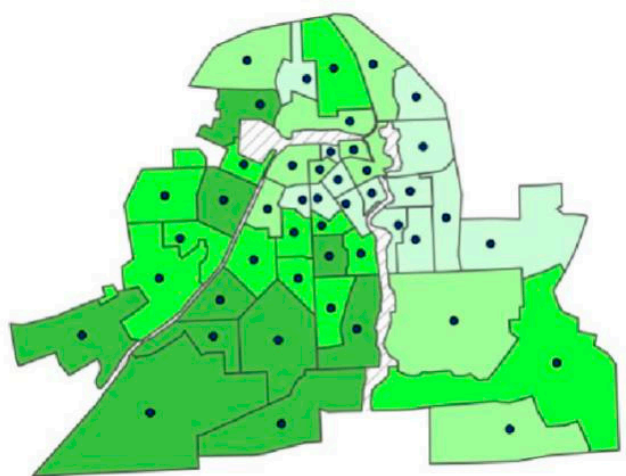

a

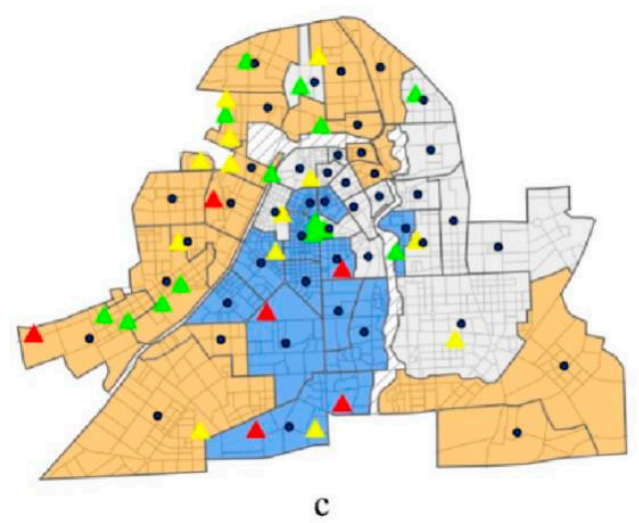

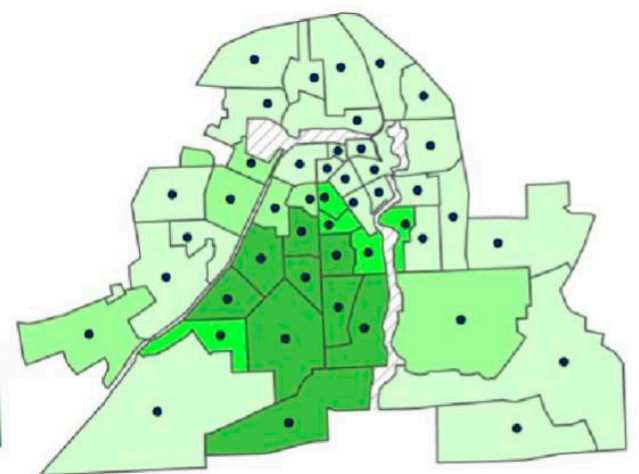

$\mathrm{b}$

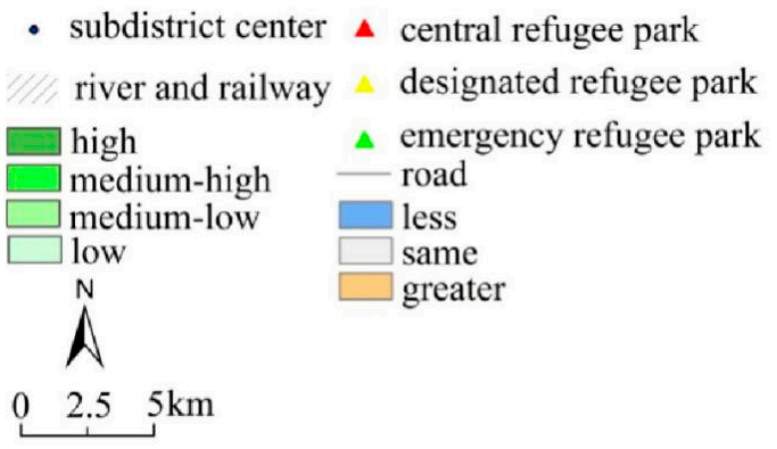

Figure 4. Comparison of evaluation results between the nearest neighbor method and the Gaussian-based two-step floating catchment area method. (a) and (b) are classes of green space per capita from the nearest neighbor method and the Gaussian-based two-step floating catchment area method, respectively, and (c) is the differences of green space per capita between the two methods.

\subsection{Evaluation of Spatial Layout of Refugee Parks}

Among the 33 refugee parks located in Changchun, 15 parks are characterized by a population accommodation capacity of more than 1, and accounted for $45.5 \%$ of the refugee parks. Almost half of the refugee parks can satisfy the per capita refugee needs of the population within their service extent. The average population accommodation capacity is 1.5. The minimum population accommodation capacity equals 0.1 for a total of five refugee parks. The maximum accommodation capacity of 7.7 is found in the Automobile Theme No. 8 refugee park, in the southwest-central urban area. Therefore, the maximum population accommodation capacity is 77 times higher than the minimum population accommodation capacity, with a standard deviation of 1.87 (Table 2).

Among the six central refugee parks, there are five parks with a population accommodation capacity more than 1, accounting for $83.3 \%$. Most of the central refugee parks can satisfy the per capita refugee needs of the population within their service extent. The average population accommodation capacity equals 3.9. The minimum population accommodation capacity of 0.9 was determined for the No. 17 refugee park, located in the core of the central urban area, whereas the maximum population accommodation capacity was found to be Automobile Theme park. As such, the maximum population accommodation capacity is 8.6 times higher than the minimum population accommodation capacity, with a standard deviation of 2.2 for central refugee parks.

Among the 13 designated refugee parks, there are only five parks with a population accommodation capacity of more than 1, accounting for $38.5 \%$. Few designated refugee parks can satisfy the per capita refugee needs of the population within their service extent. The average population accommodation capacity is 0.9 . The minimum population accommodation capacity equals 0.1 for the No. 22 and No. 23 refugee parks, located at the eastern border of the central urban area. The No. 20 refugee park 
is characterized by the maximum population accommodation capacity of 2.8 and is located in the southern-central urban area. As can be seen, the maximum population accommodation capacity is 28 times higher than the minimum population accommodation capacity, with a standard deviation of 0.7 .

Table 2. The population accommodation capacity of refugee parks in the central area of Changchun.

\begin{tabular}{|c|c|c|c|c|}
\hline Park IDs and Name & & Open Space Area $\left(\mathrm{hm}^{2}\right)$ & Service Area $\left(\mathrm{hm}^{2}\right)$ & Population Accommodation Capacity \\
\hline \multirow{14}{*}{ Emergency refugee park } & 4 & 2.7 & 433.8 & 0.3 \\
\hline & 5 & 1.7 & 642.2 & 0.1 \\
\hline & 6 & 4.9 & 324.1 & 1.1 \\
\hline & 7 & 5.1 & 287.9 & 1.5 \\
\hline & 9 & 4.0 & 563.5 & 0.3 \\
\hline & 10 & 5.9 & 234.3 & 0.7 \\
\hline & 13 & 2 & 417.8 & 0.1 \\
\hline & 14 & 12.4 & 680.0 & 1.8 \\
\hline & 15 & 4 & 96.0 & 1.4 \\
\hline & 24 & 3.6 & 716.0 & 0.2 \\
\hline & 26 & 0.6 & 496.1 & 0.1 \\
\hline & 28 & 3.3 & 449.8 & 0.5 \\
\hline & 29 & 2.2 & 1144.5 & 0.3 \\
\hline & 31 & 5.5 & 32.7 & 6.6 \\
\hline \multirow{13}{*}{ Designated refugee park } & 1 & 10.4 & 517.5 & 0.6 \\
\hline & 3 & 13.94 & 709.8 & 0.4 \\
\hline & 11 & 17.2 & 507.0 & 0.3 \\
\hline & 12 & 11.5 & 213.5 & 0.5 \\
\hline & 16 & 14.7 & 323.6 & 0.5 \\
\hline & 20 & 21.6 & 517.6 & 2.8 \\
\hline & 22 & 8.4 & 5092.0 & 0.1 \\
\hline & 23 & 8.8 & 1623.3 & 0.1 \\
\hline & 25 & 23.8 & 1884.7 & 1.1 \\
\hline & 27 & 15.4 & 201.8 & 1.5 \\
\hline & 30 & 7 & 229.1 & 0.9 \\
\hline & 32 & 10.7 & 137.4 & 1.6 \\
\hline & 33 & 16.1 & 207.3 & 1.3 \\
\hline \multirow{6}{*}{ Central refugee park } & 2 & 46.3 & 248.0 & 4.0 \\
\hline & 8 & 67.9 & 416.0 & 7.7 \\
\hline & 17 & 51.8 & 758.3 & 0.9 \\
\hline & 18 & 167 & 1356.7 & 2.4 \\
\hline & 19 & 64.4 & 835.5 & 3.0 \\
\hline & 21 & 84 & 953.0 & 5.5 \\
\hline Average value & & 21.7 & 704.6 & 1.5 \\
\hline Standard deviation & & 33.4 & 882.0 & 1.87 \\
\hline
\end{tabular}

Note: Please refer to Figure 1 for park locations.

Among the 14 emergency refugee parks, there are five parks with a population accommodation capacity of more than 1, accounting for $35.7 \%$. A minority of the emergency refugee parks can satisfy the per capita refugee needs of the population within their service extent. The average population accommodation capacity is 1.1 . The minimum population accommodation capacity is 0.1 , with three parks predominantly located in the peripheral area of the central urban zone. In contrast, 
the maximum population accommodation capacity equals 6.6 and is found in the No. 31 refugee park located in the western part of the central urban area. Consequently, the maximum population accommodation capacity is 66 times higher than the minimum population accommodation capacity, with a standard deviation of 1.6.

Overall, the population accommodation capacity varies considerably among the 33 refugee parks in Changchun. The population accommodation capacity varies the most among the central refugee parks, followed by the emergency and designated refugee parks. Most of the refugee parks with a population accommodation capacity of less than 1 are located at the peripheries of the central urban area, and only some of them are situated in the core of the central urban area.

\section{Discussion}

The service extent, determined by the Gaussian-based two-step floating catchment area method, may be greater, the same as, or less than that determined by the nearest neighbor method. When a sub-district has high-grade refugee parks, elevated road network density, and high population density, the accessibility of green spaces determined by the nearest neighbor method is lower than that determined by the Gaussian-based two-step method. When a sub-district has low-grade refugee parks, insignificant road network density, and low population density, the accessibility of green spaces determined by the nearest neighbor method is higher than that determined by the Gaussian-based two-step method. When a sub-district has no refugee parks or the grade of refugee parks is opposite to the road network, and population densities, the accessibility of green spaces determined by the nearest neighbor method is similar to that determined by the Gaussian-based two-step method. In summary, the differences between the evaluation results of the two methods lie in the size of refugee parks, road network density, and population density in accordance with the results obtained by Cetin (2015) [37] and Stessens et al. (2017) [38]. Overall, the Gaussian-based two-step method, which does not consider the grade of refugee parks and uses a unified service radius, produces greater error [39,40].

We showed that the nearest neighbor method could identify the degree of unevenness of the spatial distribution of refugee parks. Among the 33 refugee parks in Changchun, nearly half of them can satisfy the per capita refuge needs of the population within their service extent. However, the spatial distribution is not even among the different grades of refugee parks. In addition, the method identified a substantial gap between the extreme values of population accommodation capacity: The maximum population accommodation capacity is 77 times as high as the minimum population accommodation capacity in the city. Most of the refugee parks that cannot satisfy the per capita refuge needs within their service extent are located at the peripheries of the central urban area, and a few of them are distributed in the core of the central urban area. This finding is consistent with the conclusions of other studies. For instance, Wu et al. (2018) [41] and Yang et al. (2015) [42] showed that the peripheries of Chinese cities are mostly low-income residential areas and have less access to parks and green spaces. The nearest neighbor method reveals a tendency to the uneven spatial distribution of refugee parks and the results can be used to provide policy suggestions to the municipal government in order to optimize the layout of urban refugee parks. We also showed that the nearest neighbor method could quantitatively estimate the relationship between the spatial layout of refugee parks and the distribution of the population. To optimize the spatial layout of refugee parks, it is necessary to consider the spatial relationship between different types of refugee parks. In the process of emergency prevention, a refuge from disasters, disaster relief, and post-disaster reconstruction, different types of refugee parks undertake different tasks and play different roles. It is vital to build a system of refugee parks with a rational hierarchy and close interrelationship. Our studies showed that in a large city like Changchun with relatively flat terrain and natural barriers, a mosaic layout pattern for different types of refugee parks is beneficial when a disaster strikes. Emergency refugee parks can be accessed by the residents within the shortest amount of time in the event of a sudden disaster. Considering the scarcity of land resources left in central urban areas of large cities, it is essential to increase the number of emergency refugee parks and open spaces. This can improve mass emergency-related transportation to refugee 
parks. Using the nearest neighbor method, we determined the relationship between the optimal size of refugee parks and the spatial distribution of residents. Accordingly, unique planning suggestions can be made in regard to the spatial layout of different types of refugee parks and the timing for their access.

Scenario analysis of refugee parks can be conducted using other methodologies [12-14]. For instance, remote sensing can acquire instant information on park location, road conditions, and population distribution. However, remote sensing technologies would be limited to map population, and more importantly, it lacks the processes to allocate population to the designated refugee parks. The buffering methods in GIS can also be readily used to delineate service extent for refugee parks. However, such methods would require fixing service radii, which ignore the existing street network and human population, both being key in the nearest neighbor method. Thus, the contribution of this study provides a methodology that fills the gap of lacking road network information in the Euclidian distance methods and lacking human dimension and proximity in actual road network distance methods [31].

Nearly all large cities in the world (e.g., Chicago, Tokyo, and Beijing) are faced with an imbalance in the spatial distribution of service extent of refugee parks [31-46]. It is important to scientifically evaluate the adequate spatial distribution of different types of refugee parks, which is key to urban environmental safety. Correct spatial distribution of different types of refugee parks in a city should satisfy the spatial demand of urban residents for shelter in the event of a disaster. This study used GIS and spatial data analysis to overcome the methodological dilemma for the evaluation of the service extent of disaster prevention green spaces based on actual road networks. Based on the service extent and population indices, the optimal spatial layout of refugee parks could be developed.

It is worth noting that the nearest neighbor method is a general method and has a number of limitations for the evaluation of the service extent of urban refugee parks $[47,48]$. For example, the hypothetical conditions used in the method are simpler than the real scenarios of disasters [49]. However, not all of the hypothetical conditions can be taken into consideration. In the calculation process, the nearest neighbor method assumes that its space is entirely open to the surrounding sub-districts. This assumption is not locally realistic. In addition, the specific requirements for the refugee park facilities vary depending on the type of disaster. Parks constructed for fire and earthquake emergencies have different requirements than parks planned for a flood emergency, which must provide open spaces at high altitudes [50]. Refugee parks for gas leakage events must be positioned far away from the points of gas leakage [51]. These limitations need to be acknowledged to improve the existing methodology and disaster specific GIS and spatial data analysis need to be developed for real-world scenarios.

\section{Conclusions}

We have applied the nearest neighbor method to evaluating the spatial layout of refugee parks in Changchun, northeast China. The method effectively matched all of the sub-districts with their nearest refugee parks. The study results showed that the service extent per refugee park determined by this method suited the main features of road networks, overrode administrative boundaries, and matched each sub-district with the nearest refugee park. We showed that determining the service domain of a refugee park considered of the effect of road network resistance and spatial distribution of population as encapsulated in sub-districts. The results of the nearest neighbor method aligned with the refuge needs of the residents in case of a disaster, and they suited the real scenarios of an emergency refuge situation better.

Author Contributions: Conceptualization, X.L., C.X. and H.S.H.; Data curation, C.X.; Formal analysis, X.L.; Investigation, X.L., C.X., and H.S.H.; Methodology, Y.W.; Software, Y.W. All authors have read and agreed to the published version of the manuscript.

Funding: This work was supported by the National Natural Science Foundation of China (No. 41801108, No. 41971202, and No. 41871162), the Key Laboratory of Regional Sustainable Development Modeling, Institute of Geographic Sciences and Natural Resources Research, Chinese Academy of Sciences, and University of Missouri GIS Mission Enhancement Program. 
Conflicts of Interest: The authors declare no conflict of interest.

\section{References}

1. Adger, W.N. Vulnerability. Glob. Environ. Chang. 2006, 16, 268-281. [CrossRef]

2. Kar, B.; Hodgson, M.E. A GIS-based model to determine site suitability of emergency evacuation shelters. Trans. GIS 2008, 12, 227-248. [CrossRef]

3. Lee, Y.L.; Ishii, H.; Tai, C.A. Earthquake shelter location evaluation considering road structure. In Proceedings of the 2008 Eighth International Conference on Intelligent Systems Design and Applications, Kaohsiung, Taiwan, 26-28 November 2008; Volume 1, pp. 495-497.

4. Luo, W.; Wang, F. Measures of spatial accessibility to health care in a GIS environment: Synthesis and a case study in the Chicago region. Environ. Plan. B 2003, 30, 865-884. [CrossRef]

5. Yu, J.; Wen, J. Multi-criteria satisfaction assessment of the spatial distribution of urban emergency shelters based on high-precision population estimation. Int. J. Disaster Risk Sci. 2016, 7, 413-429. [CrossRef]

6. Lei, Y. Japan Prefecture and County Wide Area Green Space Planning and Inspirations. Chin. Landsc. Archit. 2007, 5, 44-45.

7. Li, Y.T.; Su, Y.P.; Liu, R.X. Idea of urban disaster prevention park planning. Urban Plan. 2004, 71-73.

8. Kılc1, F.; Kara, B.Y.; Bozkaya, B. Locating temporary shelter areas after an earthquake: A case for Turkey. Eur. J. Oper. Res. 2015, 243, 323-332. [CrossRef]

9. Smith, C.F.; Thuman, R.; Kain, D.J.; Ward, H. Emergency communication: Case study of problematics in public discourse regarding hurricane risks and hazards. In Proceedings of the IEEE International Professional Communication Conference, Seattle, WA, USA, 1-3 October 2007; pp. 1-6.

10. Kim, Y.S.; Lee, D.G. Seismic response of support-isolated secondary structures in a multistorey structure. Eng. Struct. 1993, 15, 335-347. [CrossRef]

11. García-Ayllón, S.; Tomás, A.; Ródenas, J.L. The Spatial Perspective in Post-Earthquake Evaluation to Improve Mitigation Strategies: Geostatistical Analysis of the Seismic Damage Applied to a Real Case Study. Appl. Sci. 2019, 9, 3182. [CrossRef]

12. Dai, D. Identifying clusters and risk factors of injuries in pedestrian-vehicle crashes in a GIS environment. J. Transp. Geogr. 2012, 24, 206-214. [CrossRef]

13. Pulugurtha, S.S.; Krishnakumar, V.K.; Nambisan, S.S. New methods to identify and rank high pedestrian crash zones: An illustration. Accid. Anal. Prev. 2007, 39, 800-811. [CrossRef]

14. Chen, P.C.; Dokic, T.; Stokes, N.; Daniel, W.G.; Mladen, K. Predicting weather-associated impacts in outage management utilizing the GIS framework. In Proceedings of the 2015 IEEE PES Innovative Smart Grid Technologies Latin America (ISGT LATAM), Montevideo, Uruguay, 5-7 October 2015; pp. 417-422.

15. Fernandez, A.R.; Studnek, J.R.; Margolis, G.S. Disaster preparedness of nationally certified emergency medical services professionals. Acad. Emerg. Med. 2011, 18, 403-412. [CrossRef]

16. Wolshon, B. Emergency transportation preparedness, management, and response in urban planning and development. J. Urban Plan. Dev. ASCE 2007, 133, 1-2. [CrossRef]

17. Ye, C.; Hu, L.; Li, M. Urban green space accessibility changes in a high-density city: A case study of Macau from 2010 to 2015. J. Transp. Geogr. 2018, 66, 106-115. [CrossRef]

18. Dai, D. Racial/Ethnic and socioeconomic disparities in urban green space accessibility: Where to intervene? Landsc. Urban Plan. 2011, 102, 234-244. [CrossRef]

19. Yuen, J.W.M.; Chang, K.P.; Wong, F.K.Y. Influence of urban green space and facility accessibility on exercise and healthy diet in Hong Kong. Int. J. Environ. Res. Public Health 2019, 16, 1514. [CrossRef]

20. Wu, W.J.; Zhu, S.Y.; Zhang, W.Z. Optimal allocation of emergency shelter facilities in Beijing. Human Geogr. 2010, 25, 41-44.

21. Jiang, B.; Claramunt, C.; Batty, M. Geometric accessibility and geographic information: Extending desktop GIS to space Syntax. Comput. Environ. Urban Syst. 1999, 23, 127-146. [CrossRef]

22. Fang, Z.; Li, Q.; Li, Q. A proposed pedestrian waiting-time model for improving space-time use efficiency in stadium evacuation scenarios. Build. Environ. 2011, 46, 1774-1784. [CrossRef]

23. Dou, K.; Zhan, Q. Accessibility analysis of urban emergency shelters: Comparing gravity model and space syntax. In Proceedings of the International Conference on Remote Sensing, Environment and Transportation Engineering, Nanjing, China, 24-26 June 2011; pp. 5681-5684. 
24. Cheng, L.; Wang, F.H.; Xiu, C.L. Trade area of supermarket in Changchun central urban area based on GIS. Econ. Geogr. 2014, 34, 54-58.

25. Alcada-Almeida, L.; Tralh, O.L.; Santos, L. A Multiobjective approach to locate emergency shelters and identify evacuation routes in urban areas. Geogr. Anal. 2009, 41, 9-29. [CrossRef]

26. Bai, L.M.; Xiu, C.L.; Feng, X.H. Ecological risk assessment for Changchun based on land use change. Ecol. Sci. 2019, 38, 26-35.

27. Population Stat World Statistical Data. Changchun, China Population. Available online: https: //populationstat.com/china/Changchun (accessed on 5 July 2020).

28. Du, X.; Jin, X.; Yang, X. Spatial-temporal pattern changes of main agriculture natural disasters in China during 1990-2011. J. Geogr. Sci. 2015, 25, 387-398. [CrossRef]

29. Changchun Municipal People's Government. Changchun's Comprehensive Disaster Prevention and Mitigation Plan (2016-2020). Released by the General Office of Changchun Municipal People's Government. Available online: http://www.changchun.gov.cn/zw/xxgk/gkzl/cczhengbao/2018/d05qzb_5152/szfbgtwj_5210/ 201808/t20180813_454356.html (accessed on 5 July 2020).

30. Hao, F.L.; Shi, X.; Bai, X.; Wang, S.J. Geographic detection and multifunctional land use from the perspective of urban diversity: A case study of Changchun. Geogr. Res. 2019, 38, 247-258.

31. Li, X.L.; Xiu, C.L.; Cheng, L. Evaluation on spatial structure and rationality of city parks based on goal of disaster prevention: An application of proximal area method in Changchun, China. Chin. J. Appl. Ecol. 2016, 27, 3641-3648.

32. Masuda, N. Disaster Refuge and Relief Urban Park System in Japan. Landsc. Archit. Front. 2014, 2, 52-60.

33. Lin, Y.C. Study on Planning of Urban Disaster-Prevention Park: A Case of the Disaster-Prevention Theme Park in Taipei City; Architecture \& Building Research Institute, Ministry of Interior: Taipei, Taiwan, 2007; pp. 4-5, 21-22.

34. Yuan, Y.; Ren, X.S. Discussion on planning and construction of disaster prevention park in Shanghai city. J. Disaster Prev. Mitig. Eng. 2010, 30, 452-458.

35. Zhu, Y.; Wang, H.; Zan, S.P. Countermeasures of green space construction of disaster-prevention parks in Urumqi. Urban Plan. 2009, 33, 48-52.

36. Wei, Y.; Xiu, C.1.; Gao, R. Evaluation of green space accessibility of Shenyang using Gaussian based 2-step floating catchment area method. Prog. Geogr. 2014, 33, 479-487.

37. Cetin, M. Using GIS analysis to assess urban green space in terms of accessibility: Case study in Kutahya. Int. J. Sustain. Dev. World Ecol. 2015, 22, 420-424. [CrossRef]

38. Stessens, P.; Khan, A.Z.; Huysmans, M. Analysing urban green space accessibility and quality: A GIS-based model as spatial decision support for urban ecosystem services in Brussels. Ecosyst. Serv. 2017, 28, 328-340. [CrossRef]

39. Fan, P.; Xu, L.; Yue, W. Accessibility of public urban green space in an urban periphery: The case of Shanghai. Landsc. Urban Plan. 2017, 165, 177-192. [CrossRef]

40. Hashtarkhani, S.; Kiani, B.; Bergquist, R. An age-integrated approach to improve measurement of potential spatial accessibility to emergency medical services for urban areas. Int. J. Health Plan. Manag. 2020, 35, 788-798. [CrossRef] [PubMed]

41. Wu, J.; He, Q.; Chen, Y. Dismantling the fence for social justice? Evidence based on the inequity of urban green space accessibility in the central urban area of Beijing. Environ. Plan. B Urban Anal. City Sci. 2020, 47, 626-644. [CrossRef]

42. Yang, J.; Li, C.; Li, Y. Urban green space, uneven development and accessibility: A case of Dalian's Xigang District. Chin. Geogr. Sci. 2015, 25, 644-656. [CrossRef]

43. Le, T.M.; Schiel, K.; Caruso, G. The provision of urban green space and its accessibility: Spatial data effects in Brussels. PLoS ONE 2018, 13, e0204684.

44. Miller, S. Greenspace volunteering post-disaster: Exploration of themes in motivation, barriers, and benefits from post-hurricane park and garden volunteers. J. Environ. Plan. Manag. 2019, 1-18. [CrossRef]

45. Zepp, H.; Groß, L.; Inostroza, L. And the winner is? Comparing urban green space provision and accessibility in eight European metropolitan areas using a spatially explicit approach. Urban For. Urban Green. 2020, 49, 126603. [CrossRef]

46. Takayuki, N.; Takahiro, A. Policy Changes in Green Space Preparation of Harbors and Survey of Prosperous Site Preparation in the Port of Yokohama. Collect. Artic. JSCE B3 Ocean Dev. 2019, 75, 1953-1958. 
47. Finley, A.O.; Datta, A.; Cook, B.D.; Morton, D.C.; Andersen, H.E.; Banerjee, S. Efficient algorithms for Bayesian nearest neighbor Gaussian processes. J. Comput. Graph. Stat. 2019, 28, 401-414. [CrossRef] [PubMed]

48. Rumaling, M.I.; Chee, F.P.; Dayou, J.; Hian Wui Chang, J.; Soon Kai Kong, S.; Sentian, J. Missing Value Imputation for PM 10 Concentration in Sabah using Nearest Neighbour Method (NNM) and Expectation-Maximization (EM) Algorithm. Asian J. Atmos. Environ. 2020, 14, 1-11. [CrossRef]

49. Ting, K.M.; Zhu, Y.; Carman, M.; Zhu, Y.; Washio, T.; Zhou, Z.H. Lowest probability mass neighbour algorithms: Relaxing the metric constraint in distance-based neighbourhood algorithms. Mach. Learn. 2019, 108, 331-376. [CrossRef]

50. Plate, E.J. Flood risk and flood management. J. Hydrol. 2002, 267, 2-11. [CrossRef]

51. Fraiwan, L.; Lweesy, K.; Bani-Salma, A.; Mani, N. A wireless home safety gas leakage detection system. In Proceedings of the 2011 1st Middle East Conference on Biomedical Engineering, Sharjah, United Arab Emirates, 21-24 February 2011; pp. 11-14.

(C) 2020 by the authors. Licensee MDPI, Basel, Switzerland. This article is an open access article distributed under the terms and conditions of the Creative Commons Attribution (CC BY) license (http://creativecommons.org/licenses/by/4.0/). 